\title{
Author Correction: Vertical transmission of sponge microbiota is inconsistent and unfaithful
}

Johannes R. Björk (DD, Cristina Díez-Vives, Carmen Astudillo-García, Elizabeth A. Archie and José M. Montoya (iD

Correction to: Nature Ecology \& Evolution https://doi.org/10.1038/s41559-019-0935-x, published online 8 July 2019.

In the version of this Article originally published, the following sentence was mistakenly omitted from the end of the Acknowledgements section: 'This project also received funding from the European Union's Horizon 2020 research and innovation programme under the Marie Skłodowska-Curie grant agreement No. 796011.' This has now been corrected.

Published online: 2 March 2021

https://doi.org/10.1038/s41559-021-01419-x

(c) The Author(s), under exclusive licence to Springer Nature Limited 2021 